PS10.07.23 STRUCTURAL INVESTIGATIONS OF MICROWAVE TITANATE CERAMICS. W. Wong-Ng, A. R. Drews, T. A. Vanderah, R. S. Roth, L. A. Bendersky, Materials Science and Engineering Laboratory, National Institute of Standards and Technology, Gaithersburg, MD 20899

Dielectric ceramics are used extensively in the wireless communications industry. Very high dielectric constant ( $\mathrm{k}$ ) materials with low dielectric loss are of interest because of the possibility of miniaturization of electrical components. However, some high- $\mathrm{k}$ materials have large temperature coefficients $\left(T_{W}\right)$ of the dielectric constant under typical operating conditions (e.g. $\mathrm{Sr}_{\mathrm{TiO}}$ ). Therefore, phases with improved properties, or phases that exist in equilibrium with $\mathrm{SrTiO}_{3}$ which have $\mathrm{T}_{k}$-values with the opposite sign could be useful in producing multiphase materials with a zero overall $\mathrm{T}_{\mathrm{K}}$ and have promising properties for the development of advanced microwave resonators.

We have examined numerous compositions in preparing the phase diagrams for the $\mathrm{BaO}-\mathrm{Fe}_{2} \mathrm{O}_{3}-\mathrm{TiO}_{2}$ and $\mathrm{SrO}-\mathrm{Ti}_{2} \mathrm{O}_{3}-\mathrm{Nb}_{2} \mathrm{O}_{5}$ systems. These diagrams provide processing-map information for modifying the properties of important ceramics. In the course of this investigation, we encountered a number of new compounds. A total of 16 ternary compounds were found in the $\mathrm{BaO}-\mathrm{Fe}_{2} \mathrm{O}_{3}-\mathrm{TiO}_{2}$ system and at least 4 in the $\mathrm{SrO}-$ $\mathrm{Ti}_{2} \mathrm{O}_{3}-\mathrm{Nb}_{2} \mathrm{O}_{5}$ system. In this report, we describe the results of structural studies of various compounds found in these systems.

PR10.07.24 THE USE OF ELECTRON CRYSTALLOGRAPHY TO DETERMINE THE STRUCTURAL DEPENDENCE OF NONLINEAR OPTICAL PROPERTIES IN ORGANIC MOLECULES. Z.X. Zhang, C. Gilmore* U. Kolb, I. G. Voigt-Martin, Institut fur Physikalische Chemie der Universitat Mainz, Jakob welder weg 11, D-55099 Mainz, Germany, "Department of Chemistry University of Glasgow, Glasgow G12 8QQ Scotland, UK

4Dimethylamino-3-Cyanobiphenyl was characterized with respect to linear and non-linear optical properties in the crystal as well as solution. The crystal structure was completely solved by a combination of electron diffraction and computer modelling as well as an ab initio determination using maximum entropy combined with log likelihood statistics. From this model $\mu$ and static second order polarisability $\beta$ were calculated. Their relevance to the values obtained by a combination of polarisation dependent measurements of Electric Field Induced Second Harmonic Generation (EFISH) and Hyper Rayleigh Scattering (HRS) in solution are discussed.

The molecular second order hyperpolarisability tensor was found to be dominated by one single component. The orientations of the dipole and the vectorial parts of the second order polarisability allow a deeper insight into the non-linear optical properties of the crystal.

PS10.07.25 SYNTHESIS, PROPERTIES AND CRYSTAL STRUCTURES OF COMPOUNDS IN THE SYSTEMS $\mathrm{KTIOPO}_{4}-\mathrm{TITIOPO}_{4}, \mathrm{RbTiOPO}_{4} \mathrm{TITIOPO}_{4} \mathrm{AND} \mathrm{KTiOPO}_{4}-$ $\mathrm{KGeOPO}_{4}$ N.I.Sorokina, D.Y.Lee, I.A. Verin, V.I.Simonov, Inst.of Crystallography RAS, Leninskii pr.59, Moscow, 11733, Russia V.I.Voronkova., V.K.Yanovsky, Moscow State U.,Dept. Of Physics, Lennkiye Gory, Moscow, 117234, Russia.

Single crystals of $\mathrm{TITiOPO}_{4}, \quad \mathrm{Rb}_{0.77} \mathrm{Tl}_{0.23} \mathrm{TiOPO}_{4}$,

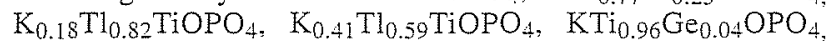
$\mathrm{KTi}_{0.94} \mathrm{Ge}_{0.06} \mathrm{OPO}_{4}, \mathrm{KTi}_{0.82} \mathrm{Ge}_{0.18} \mathrm{OPO}_{4}$ and $\mathrm{KGeOPO}_{4}$ which belongs to the group of $\mathrm{KTiOPO}_{4}$-type compounds, ferroelectric superionic conductors with nonlinear optical characteristics, were prepared by spontaneous crystallization from flux. The temperature dependence of relative dielectric constant $\varepsilon_{33}$ and electric conductivity of crystals $\sigma_{33}$ were measured at a frequency of $1 \mathrm{MHz}$ by means of the TESLA BM341E bridge. A full X-ray structural study of the above single crystals was performed on CAD-4F ENRAF-NONIUS autodiffractometer.

In the course of the structural investigation a deficiency at cationic sites was studied, the chemical formula was refined, anharmonic thermal motion of T11, T12 atoms was established and characterized.The replacement of Ti by Ge atoms gives rise to the shortening of bond lengths in Ti-octahedra and elongation of the shortest bond lengths. As a result $\mathrm{GeO}_{6}$-octahedra are much less distorted than $\mathrm{TiO}_{6}$. In the $\mathrm{KTiOPO}_{4}$ structure $\mathrm{TiO}_{6}$-octahedra are distorted to a larger extent than in sample where $\mathrm{K}$ atoms are partially or fully replaced by Tl. This is one of the structural reasons for the differences in nonlinear optical characteristics of the above crystals.

\section{Materials VIII}

\section{Powder Diffraction}

MS10.08.01 LIMITS ON PRECISION AND ACCURACY IN POWDER DIFFRACTION DATA ANALYSIS. W I F David, ISIS Science Division, Rutherford Appleton Laboratory, Chilton, Didcot, Oxon OX11 0QX, U.K.

Powder diffraction is a powerfut technique for the elucidation of increasingly complicated crystal structures. The present generation of high resolution X-ray and neutron powder diffractometers permits not only the refinement but also the ab-initio structure solution of complex inorganic materials, such as zeolites, and organic compounds, such as small diug structures with up to 30 independent non-hydrogen atoms. Indeed there is a belief that more intensity and more resolution will inevitably lead to the determination of more complex crystal structures from powder data. This talk will address the question of the limits of precision, accuracy and complexity available with current and next-generation diffractometers. The differences between precision and accuracy will be highlighted; situations where precision alone is necessary will be illustrated. Systematic errors will be shown to be the main source of loss of accuracy and emphasis will be stressed on care of sample preparation and the use of correct physical models to describe both samples and instrumental contributions to the diffraction profile. Neutron powder diffraction, with its intrinsically fewer systematic errors will be shown to offer the most accurate results. Optimised data collection methods for $\mathrm{X}$ ray diffraction will be described that attempt to minimise systematic errors and produce precise results.

MS10.08.02 DATA COLLECTION, ANALYSIS AND ACCURACY IN SYNCHROTRON X-RAY POWDER DIFHRACTION. D. E. Cox, Physics Department, Brookhaven National Laboratory, Upton, NY 11973, USA

As more and more dedicated high-resolution powder diffraction beamlines become operational at synchrotron $x$-ray sources and the number of users continues to grow, it is increasingly important to optimize procedures for data collection and analysis, to minimize systematic errors such as inadequate powder randomization, preferred orientation and microabsorption, and to improve the overall level of accuracy. Prior to data collection, careful consideration must be given to specimen preparation, diffraction geometry, choice of wavelength and counting statistics. The peak profiles should be carefully examined for anisotropic sample broadening effects and lattice distortions; pattern fitting by the LeBail method [1] is strongly recommended at this stage, especially since the resulting profile parameters can then be fixed. During stuucture refinement, an attempt must be made to judge whether the introduction of additional structural parameters results in a significant improvement to the fit.

A summary will be given of some of the lessons learned at beamline $\mathrm{X} 7 \mathrm{~A}$ at the Brookhaven National Synchrotron Light Source during the past ten years. Some emphasis will be given to reference standards, data collection with capillary specimens, the use of short wavelengths $(\leq 0.7 \AA)$, absorption corrections, anisotropic peak-broadening, the accuracy of structural parameters and occupancy factors for light atoms, and significance tests.

[1] A. Le Bail, H. Duroy and J. L. Fourquet, Mater. Res. Bull. 23, 447 (1988).

This work was supported by the US Department of Energy, Division of Materials Sciences, under contract no. DE-AC02-76CH00016. 